\title{
Evaluating Qualico: An Ontology to Facilitate Qualitative Methods Sharing to Support Open Science
}

Julian Hocker ( $\square$ julian.hocker@dipf.de )

Leibniz Institute for Research and Information in Education: DIPF Leibniz-Institut fur Bildungsforschung und Bildungsinformation https://orcid.org/0000-0003-3417-9486

\section{Taryn Bipat}

University of Washington

\section{David W. McDonald}

University of Washington

\section{Mark Zachry}

University of Washington

\section{Research}

Keywords: ontology evaluation, open science, qualitative coding

Posted Date: January 20th, 2021

DOI: https://doi.org/10.21203/rs.3.rs-148261/v1

License: (9) This work is licensed under a Creative Commons Attribution 4.0 International License. Read Full License

Version of Record: A version of this preprint was published at Journal of Internet Services and Applications on August 9th, 2021. See the published version at https://doi.org/10.1186/s13174-02100135-w. 


\title{
RESEARCH
}

\section{Evaluating QualiCO: An ontology to facilitate qualitative methods sharing to support open science}

\author{
Julian Hocker ${ }^{1}$, Taryn Bipat ${ }^{2}$, David W. McDonald ${ }^{2}$ and Mark Zachry ${ }^{2}$
}

\footnotetext{
${ }^{*}$ Correspondence:

julian.hocker@dipf.de

${ }^{1}$ DIPF — Leibniz Institute for Research and Information in

Education, Frankfurt am Main, Germany

Full list of author information is

available at the end of the article
}

\begin{abstract}
Qualitative science methods have largely been omitted from discussions of open science. Platforms focused on qualitative science that support open science data and method sharing are rare. Sharing and exchanging coding schemas has great potential for supporting traceability in qualitative research as well as for facilitating the re-use of coding schemas. In this study, we describe and evaluate QualiCO, an ontology for qualitative coding schemas. QualiCO is designed to describe a wide range of qualitative coding schemas. Twenty qualitative researchers used QualiCO to complete two coding tasks. In our findings, we present task performance and interview data that focus participants' attention on the ontology. Participants used QualiCO to complete the coding tasks, decreasing time on task, while improving accuracy, signifying that QualiCO enabled the reuse of qualitative coding schemas. Our discussion elaborates some issues that participants had and highlights how conceptual and prior practice frames their interpretation of how QualiCo can be used.
\end{abstract}

Keywords: ontology evaluation; open science; qualitative coding

\section{Introduction}

The basic ideas behind open science are probably centuries old and are present in the earliest scientific letters and in the establishment of journals for sharing research results. The expansion of science as a productive discipline, and concerns about junk science, have generated renewed interest in the concept of open science. Since roughly 2000, discussions of what constitutes open science have grown [1]. Some clear components of open science include the sharing of scientific data, sharing and explaining scientific methods, as well as sharing research results.

For many open science efforts the accessibility of data is considered the primary means of openness across multiple fields of knowledge, people, and institutions as well as the validation of results. According to the Open Science Collaboration (OSC), prior research "should not gain credence because of the status of authority of their originator but by the replicability of their supportive evidence" [2]. In the midst of the ongoing replication crisis it is important to be able to have access to prior data as well as clearly described methodologies for collecting, cleaning, manipulating and analyzing such data. Furthermore, sharing data allows researchers to build off the assumptions and efforts of past research. Access to open data enables the broadening of research scope and the ability to diversify perspectives in science [3]. 
A focus on open data as a road to open science has resulted in concerns about potential unintended outcomes and the differential benefits for scientists of different status [4]. Addressing these challenges is important for facilitating widespread adoption of open science data sharing. However, focusing on data sharing to the exclusion of other, equivalent challenges faced by different scientific traditions may be short sighted. The sharing of methods complements the sharing of data, making all the research process transparent and allowing for traceability and reproducability of research.

To date, the most of the focus on data and methods sharing in open science has been in the quantitative sciences. Scientific disciplines that rely on qualitative methods, in contrast, have largely been overlooked or completely omitted from broad discussions of open science. In scientific disciplines that use qualitative methods, researchers tend to focus on reliability and traceability of data and methods rather than on generalizability and repeatability.

\section{The Potential for Open Science in Qualitative Methods}

A potential issue in addressing open science concerns for qualitative methods is that even within that term of art, the range of methodological approaches is quite broad. Given the range of methods, some researchers have rejected the idea of even attempting to define qualitative research [5]. Others who attempt definitions have offered that qualitative research is non-numeric [6] and unstructured; or, simply it is defined as the "opposite" of quantitative research.

The juxtaposition of qualitative methods in contrast to quantitative methods is aligned with the contrasting underlying philosophical stances of interpretivism and positivism. But this simplification obscures the breadth within qualitative traditions; within qualitative methods there is a range of interpretivism to positivism. Some ethnographic methods approach data and their explanation as a purely interpretivist act, seeking to describe human experiences in ways that can be more broadly understood. At the more positivist end of the qualitative method spectrum we find systematic qualitative coding methods. In such systematic methods researchers are data-driven, looking for repeatable, systematic ways to analyze or code the data.

Another way of describing methods that do coding can be found in German research literature. This literature distinguishes between two methods for coding data: Grounded Theory and Qualitative Content Analysis. These methods also differ in the way they treat coding schemas. In Grounded Theory, coding schemas can be seen as the result of the inductive coding process where codes are generated from the data. Meanwhile, in Qualitative Content Analysis, coding schemas can be seen as a tool that tells the researchers how they should code which parts of the texts. However, within these methods there are variations, especially when it comes to developing a coding schema using a Qualitative Content Analysis; this can be done inductively, deductively or as a mixture of both. [7]

In the same way that current open science and open data platforms have not addressed all challenges of quantitative science traditions, it seems misguided to believe that all qualitative traditions could be easily supported by a single system. In fact, attempting to address the needs of qualitative researchers by building upon 
what we already know about open science might best address the needs of qualitative researchers who use methods that are closer to the positivist end of the spectrum. And that is largely the approach taken in our work here.

In this study, we describe and evaluate the ontology for qualitative coding schemas QualiCO [8]. QualiCO is designed to describe a wide range of qualitative coding schemas. In this case a qualitative coding schema is a set of codes, possibly hierarchical, that can be applied to qualitative data by a researcher. A researcher sharing a coding schema would instantiate the ontology with a designated set of codes and code descriptions. The ontology can be elaborated with sample data, publications, and various other metadata information that can make the schema more usable. We describe this more fully below.

Broadly, we believe an ontology for sharing qualitative coding schemas has the potential to make qualitative research more transparent as well as encouraging researchers to share more of their work, making their scientific process more visible. The ontology can make the reuse of qualitative schemas easier by providing a richer context for understanding the schema, how it can be applied, and for which types of data it is effective. However, as our analysis illustrates, for such a system to facilitate open science, the costs of participation need to be integrated into the workflow of researchers at a minimal cost to encourage sharing.

\section{Research Goal}

In this study, we seek to understand the performance of QualiCO, a system that was described in a prior research project focused on defining qualitative coding schemas [8]. A qualitative coding schema is the description of the qualitative codes, which can be seen as a result of the work in grounded theory or as a tool for qualitative coding [7]. QualiCO was implemented into a prototype using Semantic MediaWiki ${ }^{[1]}$ allowing researchers to add coding schemas following the standards of QualiCO. The goal of this research was to see whether a prototype using QualiCO fulfills the requirements for use in open science. More specifically, we ask:

1 How does researcher performance change using the ontology across different coding schemas?

2 Does a nearly complete instantiation of the ontology provide enough metadata for a researcher to reliably apply the coding schema?

3 How can this ontology be improved to make the reuse of coding schemas easier?

In this paper, we first outline prior work related to how open science researchers share qualitative coding schemas. We then review various ontology evaluation methodologies and describe the ontology being evaluated in this study. Following this, we outline our evaluation methods. In our findings we present task performance data and feedback on the ontology that resulted from a semi-structured interview designed to focus participants' attention on the ontology. Finally, we close by discussing the results and recommendations for what a future platform for sharing coding schemas might look like.

${ }^{[1]}$ https://www.semantic-mediawiki.org 


\section{Prior Work}

In our overview of related literature, we focus first on the general sharing of research data in open science and then on the sharing of qualitative methods. This prior work points to research procedures as well as standards for metadata that can be built upon. As we review this prior work, it is worth noting that we include as a starting point sources that are primarily about data sharing in open science because they are somewhat related but that our real focus is on sharing qualitative coding schemas. This aspect of sharing is more clearly related to methods than to the broader concern of sharing data. We thus begin with important findings related to research data sharing to consider what they may imply about method sharing. Following this, we describe the state of method sharing in qualitative research as well as archiving qualitative coding schemas.

\subsection{Data sharing and reusing in absence of method sharing}

In the last few years, several studies examining how open science researchers share their data have been reported $[9,10,11,12]$. Many of these studies notably focus on why researchers do not share their data. When researchers do share data, a major challenge is describing the data. Data description is necessary for information experts to develop adequate insights into the prior research. For this reason, researchers inclined to share their data have need to make it available via easy-touse platforms $[13,14]$. Understanding how data is input into such platforms is also important. To make shared coding schemas possible, those schemas will need to be structured following standards that can be encoded into easy-to-use platforms.

When it comes to sharing of reserach data, there have been also several advances: the FAIR principles has undergone several developments [15] as well as have the platforms that embody CARE principles [16]. The FAIR principles, which address findability, accessibility, interoperability and reuseability, formulate criteria for research data to be shared within a platform. The CARE principles are designed to support collective benefit, authority to control, responsibility and ethics. These principles offer a blueprint for the creation of research data centers, providing guiding principle for the creation of new platforms in open science. With the development of QualiCO, we addressed especially the criteria of findability for a future platform.

To begin to address such obstacles, researchers examining the work of open science argue that documentation of resources is key. For example, documentation of data is a key factor in the potential reuse of that data. Kowalczyk, S., \& Shankar, K. [17] define this needed documentation as context:"[...] context documents how datasets fit into their physical and technical environments (file formats and field descriptors) as well as into the scientific environment (experiment treatments and applications)." Faniel, et al. further demonstrate that metadata quality is important for the satisfaction of social scientists with data portals [18]. Curty [19] identified a set of factors that motivate data reuse in the social sciences: 1) the potential expansion of knowledge via re-analysis of data, 2) it is cheaper to reuse data than generate new data, and 3) the pre-endorsement that existing data is perceived to be of high quality when researchers took the time to provide it for other researchers. Factors that enable data sharing are the availability of documentation, availability of data repositories, contact with primary investigators, support from the research 
data centre as well as skills for analyzing data. Our exploratory study does not include measures comparing qualitative and quantitative research. We do see all three main factors as well in the sharing of qualitative coding schemas. We focus on the point of documentation of the coding schemas, which was also named as important for the sharing of data.

Building from these premises, we hypothesize that for the sharing of coding schemas, the quality of the provided metadata will be crucial and depends on the ontology that is used to describe the coding schemas. The underlying ontologies describe the rules for documenting coding schemas. Based on this approach, we use the definition of metadata or ontologies as "a formal, explicit specification of a shared conceptualization" [20]. Such metadata is captured in the ontological approach we describe below. It is an approach we believe has the potential to fill the gap that must be addressed if qualitative coding will be understood well enough to fulfill its potential in open science.

\subsection{Qualitative coding schemas in the context of open science}

Many projects using qualitative methods can also be considered a part of "small science" [21]. Small science is most often conducted by single researchers without published standards or platforms, yielding "dark data" [22], that is data that is functionally impossible to reuse. A part of this reuse challenge is related to limitations in how coding schemas are (or are not) shared.

In qualitative social research, an ongoing discussion focuses on the quality of the research. An important part of this discussion focuses on descriptions of data analysis and sampling [23], [24]. Our investigation here builds upon our prior research [25], describing the development of QualiCO as well as first results of an evaluation. Those first results showed that it is important for the acceptance of a future platform that it fits the desired workflow of researchers.

Our research is specifically focused on the use of qualitative coding schemas in the work of open science. Broadly speaking, qualitative coding schemas are used with different methods, including Grounded Theory [26], general classification [27], and content analysis [28] and [29]. Following these methods, coding schemas include the codes used within the study as well as documentation about when and how to use them. Coding schemas thus include essential documentation integral to their use within the qualitative method. Sharing qualitative coding schemas, consequently, entails method sharing in open science qualitative research.

Notably, the sharing of methods is different from the sharing of data since there is more documentation needed to understand how the researchers worked using their method. In open science data sharing, the important information is the data itself and its origin. For sharing coding schemas, however, it is important to know contextual background information about the research, such as the theoretical assumptions of the researchers.

\subsubsection{Method sharing and re-using in qualitative research}

We see the sharing of coding schemas as the sharing of methods. In quantitative methods, sharing is fairly easy, typically just involving the naming of the statistical method used, e.g., linear regression plus parameters in order to show the reader what 
a researcher did in her paper. However, in qualitative research method sharing is way more complicated. Saldaña (2015) [27] shows 30 methods of coding, divided into several categories. This shows the broad range of qualitative coding methods. Added to this, qualitative coding also involves a lot of interpretation, which means that just naming your coding might not make transparent how you coded and why you came up with certain categories. If you perform deductive coding by generating codes from a model, this is easier. However, with inductive coding or mixtures of both, things are less clear.

To consider qualitative coding schemes in the context of open science is thus an inherently challenge from the onset. A known systemic obstacle in the work of open science is that research is hampered by a lack of standards for sharing research materials, including codebooks [30]. Furthermore Aguinis \& Solarino name twelve criteria for making a qualitative study reproducible, which include the kind of qualitative method, Data coding and first-order codes as well as data analysis and second - or higher-order codes [31].

This call is echoed by others who encourage the sharing of intermediate steps of research in open science, such as Grubb and Easterbrook's article [32], which explicitly addresses the "various mechanisms" used for sharing work.

Another paper names several practices that allow qualitative research to be more open: pre-registration of research projects, methodological explanations, annotation, export of codes from QDA-software as well as the sharing of data [33].

The new publication guidelines of APA [34] calls for more important information including:

- Description of selection of participants as well as the relationship to them and how long data collection (e.g., interviews) took

- Description of data analysis, which method was used, whether coding was done inductive or deductive

- Methodological process, description how researchers came to their conclusions

- Results should be described as concrete as possible, using also graphs describing the most important codes.

Summing, up we can see that the description of the data analysis is an important part of an open science practice for qualitative research. For researchers using methods like qualitative coding or Grounded Theory, the coding schema includes most of this information. Therefore it makes sense to make qualitative coding schemas more publicly available.

\subsubsection{Archiving Qualitative Coding Schemas}

Overall, we can see that the issue of data sharing fosters tension within qualitative methods, but the sharing of methods has not yet been so controversial. However, in the discussion about archiving qualitative coding schemas, there has been some development leads by the group $\mathrm{REFI}^{[2]}$. REFI took one step towards the sharing of qualitative coding schemes by creating the standard REFI-QDA Codebook[35], an exchange format with which it is possible to transfer a code in one software to the next. The readability of file format is a problem in sharing data (data sharing in sciences); however, this exchange format provides a start in order to archive and

\footnotetext{
${ }^{[2]}$ https://www.qdasoftware.org/
} 
share data. Still, different software uses different notions of coding schemas, which means that not all information is shared within the same format.

In our research, the goal is to reuse these methods. Concretely, we want to see whether the ontology QualiCO implemented into a prototype Wiki platform helps researchers reuse coding schemas for coding sample texts. In other words, we seek to discover whether researchers can reuse a method described by other researchers.

Understanding coding schemas through prior research is challenging. In some cases the creation or development of a schema is the objective of the research. For example, many who follow a Grounded Theory approach, develop coding schemas to reflect a theory of the phenomena they will ultimately attempt to describe [36]. In other qualitative research, the coding schema is a means of getting to the real result. In other words, coding schemas as they are used by qualitative researchers may be either inductive or deductive. Regardless of which approach qualitative researchers use, however, there are few systematic ways of describing and cataloging schemas and how they are applied. Consequently, meta-level discussions of qualitative coding schemas are scarce despite the widespread use of qualitative methods across fields and disciplines.

One way to facilitate the sharing of data is the creation of platforms where researchers can expose how the data was created. This would include the sharing of qualitative coding schemas, but there does not exist such a platform. There needs to be more ways of attribution towards the sharing of coding schemas in order to motivate people sharing their data. Another way to ensure this type of sharing is for journals to require the sharing of coding schemas whenever an article is published [17]. Publishing data and coding schemas together with corresponding articles would also give subsequent researchers the opportunity to easily find data, which would in turn help in the sense of expanding scientific knowledge graphs [37].

Summing up, there is currently no platform that allows the sharing of qualitative coding schemas. If such a system is to be developed, it has to fit into the workflow of the users in order to make it useful and to lower issues for uploading coding schemas to the system.

\subsection{Ontology evaluation}

Ontology evaluations have been quite diverse and ranges from technical evaluations with the focus on how the ontology supports reasoning to user-based approaches that focus on the utility or usability of the ontology. A survey by McDaniel and Story [38] categorizes ontology evaluation into five groups: Domain/Task Fit, Error Checking, Libraries, Metrics, and Modularization. Using this categorization a human centered evaluation might fall into the Metrics or the Domain/Task Fit categories.

A recent review by Palavits et al. [39] offer an overview of the most often used metrics for user studies of ontology systems. The alternative to quantitative metrics based evaluations include interviews [40], questionnaires [39] or a combination of both [41] that rely on human perceptions of the system.

Critics have argued that ontology evaluations need to be more closely aligned to their intended purposes. A Task-Driven approach as described by McDaniel and Storey [38] focuses on the goal orientation as derived from requirements engineering. 
Specifically, the goal of an ontology should be to support the information system it is built into. In our case, this means that the ontology should support the upload, search and usage of qualitative coding schemas.

Grey [42] describes this need: "An application ontology should be evaluated against a set of use cases and competency questions which represent the scope and requirements of the particular application. For example, a user query use case may contain the competency question 'what cancer cell line data is there.' This requires sufficient ontological coverage to capture the concept of 'cancer cell line'." $[42]$

The user analysis can be done using two ontologies or two versions of ontologies. Reinhold [43] evaluates an old version of the ontology against a new version, Liu et al. [44] evaluates three ontologies in order to pick one to select for a given task. The evaluation by $\mathrm{Yu}$ et al. [45] involves users in a browsing task based on the ontology in Wikipedia, which has been enriched in a different way. Another version of task-based evaluation is presented by Pittet et al. [46], where users are able to enrich the ontology,

In the development of the ontology, participatory design was used [8], therefore it made sense to focus on user-centered approaches also in the evaluation of the ontology. With this research, we present an approach to ontology evaluation using user tests. Our goal was to create a scenario for a task-driven evaluation of an ontology, wherein the users are able to use the ontology and are given a task they have to solve using the ontology.

\section{The QualiCO Ontology for Qualitative Coding Schemas}

We leverage one specific ontology designed to facilitate the sharing of qualitative coding schemas. These qualitative coding schemas are often used during systematic coding of qualitative data. Qualitative coding is performed in a range of ways, so the ontology is not aimed at any specific version. In fact, the ontology can likely support a much wider range of qualitative schema than those that we will test in the evaluation below. The ontology we use was developed through participatory design methods including interviews and observations. The participatory design methods had a diverse range of qualitative methods practitioners, but most could be described as on the positivist end of qualitative methods (c.f. Section 2). Ontology development included several rounds of feedback on prototypes that represented different aspects of the ontology [8]. This ontology has been used in one prior study that considered how it addresses specific open science challenges for qualitative methods [25].

The ontology consists of five main categories: publications, research data, study descriptions, coding schemas and codes. Each of these categories contains further information about these types of information. The following graphics shows the structure:

Figure 1 Structure of the ontology

The ontology was designed to facilitate description of qualitative schemas at differing levels of specificity. At the main level of the description we use the term 
"project" to circumscribe the schema and any artifacts that support its description. Some basic information about a schema is essential, but the ontology supports the inclusion of optional contextual information about the research activity that used or defined the ontology as well as possible research data. Naturally, not all projects will contain all potential artifacts, but the ontology was designed to represent key artifacts related to a schema. The goal of the ontology was to provide enough metadata to understand the background of the research activity as well as providing a detailed description of the coding schema. ${ }^{[3]}$

The evaluation descried in this paper is focused on the potential effectiveness of the ontology to support reuse of specific coding schemas. While any system that would use the full ontology must represent and facilitate the effective navigation of multiple projects, we will not address that specific question in this evaluation. This work focuses on the potential to reuse coding schemas.

The class "coding schemas" describes the metadata for the coding schemas contained by any project in the ontology. This includes metadata to describe the methods used for analyzing data, the theoretical background of the work, research questions as well as the process of schema creation. On a technical side, the class also supports metadata to describe any software that may have been used to create the codes. If a commercial system was used in the project the class supports upload and download of the coding schemas using the exchange format of REFI-QDA codebook $^{[4]}$ as well as REFI-QDA project ${ }^{[5]}$. In a reuse-scenario this allows researchers to get an overview of the coding schema and reuse it within a QDA-software.

The coding schema class provides a relation link to link each "code" in the schema. Each code is described using the following metadata:

- name of the code

- description of the code

- example

- counter example

- connection between codes

- provenance

- count

The fields Name of the code, Description of the code and Example are required, the other fields are optional. The ontology is designed to support systematic qualitative coding, but may support grounded theory methods as well. making connection between codes more important for Grounded theory and the number of codes more relevant for more reproducibility-oriented qualitative coding.

A prototype implementation of the ontology was built using Semantic MediaWiki and populated with two coding schemas that our research participants were able to browse. We implemented the coding schemas as well as the connections to research data, projects and publications. The research participants were able to navigate freely in the system and choose the information they needed for the specific study tasks. The MediaWiki navigation was as follows: on the main page there were links

${ }^{[3]}$ A prototype of the ontology can be found on Github https://github.com/julianhocker/ Quali-Codes-Ontology.

${ }^{[4]}$ https ://www .qdasof tware.org/products-codebook-exchange/

${ }^{[5]}$ https : //www.qdasoftware.org/products-project-exchange/ 
to overview pages for publication, study, research data and coding schema. If the participant clicked on one of these main categories, they found a page with all content within these main categories, e.g., all coding schemas in the system. From this, participants could navigate to a specific item, e.g. coding schema. On these item pages, participants found all metadata as well as links to codes, publication, study and research data, which belong to this coding schema. This navigation largely reflects the links present in the ontology diagram of Figure 1.

We set up the prototype by adding two coding schemas: one about the coding of Barnstars, which are awards in Wikipedia given to recognize specific work performed by contributors. This coding schema is described in prior work [47], [48]. The other coding schema was based on a project analyzing reviews of Amazon's voice assistant system Alexa. A version of the coding schema had been previously used to code online discussions about Sony's Aibo [49].

Both of these coding schemas have been used in prior research studies. However, while the Barnstars data had only been used for related Barnstar data, the Alexa coding schema had been previously used for a Sony Aibo study and then modified to fit comments about Amazon Alexa. This may in turn impact how the coding schema is used within the ontology because the data being coded for the Alexa task was not naturally created for that data.

Both coding schemas were also used in different ways. The Alexa schema included two coding parts, first the researcher had to find the presence of a particular anthropomorphization characteristic and then either choose whether the comment yielded an affirm or negate statement. The Barnstar task presented a hierarchical coding schema. In the coding task, the researcher first chooses a overarching code that represents the data and then chooses subcodes that fit within the larger theme. The two coding schemas were distinct making them difficult to compare directly.

Both coding schemas were described in the prototype using the QualiCO ontology. The goal in the description was to provide as much information as possible. Therefore, in the level of publication, study, research data and coding schema all metadata fields were filled out. There was also a graphical overview of the structure of the codes provided. On the coding schema for the Barnstars, for each top-level code a description and an example were provided. For the lower-level codes only the description was provided. The pictures $3,5,2$ and 4 show the instantiation:

Figure 2 Overview of metadata for codding schema for the Barnstars task.

Figure 3 Table representation of top-level codes in the task Barnstars.

Figure 4 Graphical representation of top-level codes in the task Barnstars. 
Figure 5 Detailed description of codes in the Barnstars task.

For the coding schema supporting the Alexa task, we provided a description of each code as well as examples. However, we did not provide examples for affirmation and negation for every code, though we did so for some of them. The graphics 6,7 and 8 show the instantiation of this coding schema. Compared with the Barnstar coding schema, the Alexa coding schema was more flat, which means that the participants had to keep all codes mentally active, while at the Barnstar coding schema, they only needed to have the top-level codes mentally active.

Figure 6 Overview of metadata for the Alexa coding schema.

Figure 7 Graphical representation of top-level codes in the Alexa task.

\section{Methodology}

Our goal was to understand potential user's experiences with this type of open data system, focusing on how potential users might see this type of system as part of their qualitative research process. We structured our evaluation process around a pair of qualitative coding tasks that would require that participants in our study explore and use the various portions of the system. This was used to test not only the ontology by itself, but rather test an application of the ontology. Since QualiCO will be used as an ontology for open science, we can follow the schema by [13] dividing the tasks in open science into three points: willingness to share, locating shared data, and the reuse of shared data. In this evaluation, we want to focus on the reusing of qualitative coding schemas, therefore we gave the participants tasks where they had to apply a coding schema to new data.

We recruited participants for our study through flyers, targeted mailing lists, and personal contacts at the University of Washington in Seattle. All of our participants had prior experience as a researcher or research assistant on a project that used systematic qualitative coding as part of the data analysis. While we recruited broadly at our university, all participants had a background in human-centered design or information science. Our participants spanned undergraduates, graduate students and professional research staff. Undergraduate student participants are characterized as beginners with only one hands-on project experience using systematic coding. The graduate students and professional researchers all had additional training and one or more project experiences. In total we had 20 participants complete our study; 4 undergraduates, 14 graduate students, and 2 professional research staff members.

The study was structured in several distinct phases. The first phase included an introduction to the system. The introduction consisted of a comprehensive video 
Figure 8 Detailed description of codes in the Alexa task.

tutorial that had been prepared to illustrate key aspects of the prototype and ontology. An 8 minute long video highlighted each area of the ontology, describing each area in detail and demonstrated how to navigate the prototype system. The participant was then asked to take a "system comprehension quiz," which gave the participants a chance to familiarize themselves with the system. Participants were told they could use the prototype to answer the questions in the quiz. After the participant completed the quiz, the researcher running the study went over the answers and clarified any answers that were wrong. The video tutorial and comprehension quiz helped ensure that all participants received a consistent presentation and that their basic understanding of the ontology and prototype were all at a similar level.

In the second phase, the participants were challenged to use the prototype to explore qualitative coding schemas and apply them to sample data. The prototype contained two research coding schemas for this phase of the experiment. The two schemas chosen were selected from several potential schemas that the research team had used on prior research projects. Both schemas had been applied to different data sets on at least two occasions. The versions of the coding schemas loaded into the prototype were simplified to facilitate a timely completion of the coding task, but still reflected the key analytical questions raised by the original schemas.

The schemas were applied to data as separate timed tasks and participants were allowed up to 25 minutes per task. The task order was counter-balanced to control for possible learning effects. In each task, the participant needed to identify and review one schema and apply the schema codes to four sample texts. The participant was given a paper based coding sheet with the four text samples and had to circle or write down the code(s) that best fit the sample text. Figure 9, shows one sample from one coding task. If a participant exceeded the allotted time they were to be stopped. In fact, only one participant was stopped and a majority of the participants completed each coding task well within the allotted time.

Figure 9 Example of a coding task. Participants were given instructions and two samples before beginning the task. They had the ability to freely use the system to complete all tasks.

In the third phase of our evaluation, we conducted semi-structured interviews to obtain qualitative data about our participant's experiences with the prototype and their strategies for applying the schemas. These closing interviews lasted 10-20 minutes and were structured around the following questions:

1 Can you briefly describe your strategy for applying the codes?

2 What was different about your strategy between the first coding scheme and the second?

3 What questions did you have about the coding schemes that you could not answer with the system?

4 What aspect of the system was most helpful to your work to apply the codes? 
5 What meta-data do you feel is missing from the system that would be helpful for these tasks?

6 Is there anything else you would like to share about your experience with the system?

7 Do you have any questions for us about this system or this study?

The interviews were were recorded and transcribed. We then conducted a thematic analysis of the transcripts. The research team met weekly to extract and refine the themes in the transcripts. The main findings from each participant were listed and then reviewed by all authors of the paper. The findings reflect common themes. In a prior publication [] we focused only on the qualitative analysis on the interviews using the themes that related to the open science framework by Birnholtz \& Bietz [13] . This analysis focuses on the task performance characteristics and their specific strategies for using schemas represented within this ontology.

\section{Findings}

Our findings are based on the quantitative and qualitative data that we collected during our structured trials. All of our participants should be considered experienced qualitative researchers. In particular, all met the condition that they had participated in at least one research project where they had performed a systematic qualitative coding. Many of our participants also participated in the development of one or more coding schemas during research projects. Four participants in the study met only the minimum threshold; the other 16 participants had more experience. Given our small number of participants who might be considered "novice" we do not breakout the results of the study by experience levels.

We structure the results of our study based on the performance of the participants in the two coding tasks. We provide both quantitative and qualitative data to demonstrate participants performance using the prototype and their perceptions of the ontology. The quantitative results demonstrate the time taken on each coding task and their accuracy at the coding task. Our qualitative results illustrate some of the users' perceptions of the prototype and how they approached the different coding tasks. Before we dive into the findings, we briefly explain some distinctions between our specific experiment and the way most qualitative coding tasks are performed.

\subsection{Qualitative coding in an Experimental Setting - Some Caveats}

In this study, we asked participants to participate in two coding tasks using schemas that were instantiated into our prototype. Participants were given a 25 minute time limit to use the prototype to code four data samples. Conducting the experiment in this way puts the prototype, the ontology, and our participants at a disadvantage. Like many experimental conditions, these conditions do not accurately mirror the way that qualitative coding is performed.

First, it is quite rare that one would simply pick up a qualitative coding schema and immediately start coding data. Often there is some time spent with the coding schema to try and understand something of what is to be coded, code inclusion or exclusion criteria, and boundary conditions between codes. As well, in many cases there is often some one to talk with about the codes; an advisor, collaborators, a 
trusted colleague. Even tangential discussions about a qualitative phenomena can provide insight or clarity to the concept. Our experiment did not provide for any form of in-situ clarification.

Second, it is fairly rare for researchers to apply two distinct coding schemas backto-back. Certainly, it can be the case that research supervisors may work with different individuals or teams who are applying different coding schemas. But in those cases a research supervisor is not likely to be applying the codes individually and challenged for their accuracy in code application. Applying two distinct qualitative coding schemas is a form of "switching gears" that is somewhat rare.

Perhaps a third distinction is that applying a coding schema as a timed task is quite uncommon. Most often individuals are allowed to compare, consider and reflect on the way that the codes are applied. This can include multiple passes through the data. We set a time limit of 25 minutes for exploring and applying each of the two schemas to sample data. While there was only one participant who "timed out", making the 25 minute limit appear reasonable, coding as a timed task, with no opportunity to return to the data, is not a realistic qualitative coding practice.

We state these caveats here, up front, because the disadvantages of this experimental condition may not be well understood by readers who are not familiar with qualitative coding. An alternative evaluation method, such as a full field deployment, could mitigate these disadvantages, but would introduce another set of complex evaluation challenges. In this work we focused on a more experimental approach, while recognizing the implications for qualitative practice as mentioned above.

\subsection{Time on Task}

During the study, each participant was asked to complete two coding tasks using two different schemas. These tasks were counterbalanced across the participants to control for potential learning effects, table 7.2 shows these results. Participants were given 25 minutes to complete each task. The table below presents the time on task data, measured to the minute. No participant used more than 25 minutes. The findings show that the Alexa coding task took approximately 2.5 minutes more to complete than the Barnstar task. Uniformly across both tasks, time on task was reduced from the first to second coding task.

\begin{tabular}{|l|l|l|l|}
\hline & All & First & Second \\
\hline Barnstar & 10.1 & 10.9 & 9.3 \\
\hline Alexa & 12.45 & 13.4 & 11.5 \\
\hline
\end{tabular}

Table 1 Time on task. The table indicates differences whether a task was taken first or second.

We performed a t-test to determine whether there the decrease in time from first to second coding tasks were significantly different. While the tests were not significant at $p<0.05$ levels, they were close. In particular, we point out that there were only 10 participants in each condition, making the likelihood of significance low.

\subsection{Task Accuracy}

We use a formula to calculate an accuracy score for each coding task. The accuracy scores were calculated using the formula below. 


\begin{tabular}{|l|c|c|c|c|c|c|}
\hline & \multicolumn{2}{|c|}{ Alexa } & \multicolumn{3}{|c|}{ Barnstar } \\
\hline & First & Second & p-value & First & Second & p-value \\
\hline Sample 1 & 9 & 3 & 0.03 & 1.1 & 0.8 & 0.26 \\
\hline Sample 2 & 8.6 & 3.9 & 0.07 & 1.2 & 1 & 0.39 \\
\hline Sample 3 & 7.65 & 5.2 & 0.19 & 3.4 & 2.5 & 0.09 \\
\hline Sample 4 & 9.1 & 4.9 & 0.11 & 5.1 & 4.8 & 0.35 \\
\hline
\end{tabular}

Table 2 Performance on each task based on whether it was taken first or second

$$
\text { AccuracyScore }=(\text { Codes Applied }- \text { CodesCorrect })+\text { CodesOmitted }
$$

We calculated each participants accuracy score as the sum of applying too many codes and omitted codes. We subtracted the number of correct codes from the number of codes applied and added the codes that were completely missed. A participant with an accuracy score of 0 (zero) would have a perfect score, indicating complete agreement with the prior coding of the sample data.

Our findings, shown in Table 2, reflect an increased number of incorrect responses in the Alexa coding task compared to the Barnstars coding task. This implies that the Alexa task where participants had to first select if a code applies and afterwards do a rating about affirmation or negation, was harder than the Barnstars task, which was a straightforward two-level coding schema task.

Uniformly, participants' accuracy improved from the first task to the second task (lower accuracy scores are better). This result, in conjunction with the improved times, suggest that the ontology and the specific prototype are not impeding the performance of the coding tasks. While the counterbalanced experimental design cannot completely eliminate all possible learning effects, that both time and accuracy improved suggest that there is some possible benefits in the ontology. Next, we consider insights from the semi-structured interviews.

\subsection{The need for additional metadata}

In the post-study interview, participants were asked if they felt that any metadata was missing from this instantiation of the ontology. Most participants felt that the metadata presented covered most of the information needed to completed the coding tasks. However, they did comment on a few additional needs that could help address the understandability and discoverability of the coding schemas.

Participants asked for additional examples of the codes with explanation of how the codes were applied to a specific example. P16 had difficulty understanding the examples for the Alexa coding task:

For the Alexa one, it would have been nice to have a positive and negative example, I don't know if that would have been a lot of work. Sometimes whenever I was reading the examples I had to decide what was the negative of that or the positive of that, so I feel like if I had an example of that, it would be helpful. Other metadata (scrolls through system) I cannot think of any, I think it was fairly straight forward. (P16)

Furthermore, participants wanted additional metadata to support their understanding of the coding schema. Specifically, they wanted additional contextual 
knowledge to better apply the codes. P3 asked for more domain specific knowledge related to Wikipedia:

"In some cases, especially for the Barnstars example, there is a lot of specific domain related knowledge that I did not have related to what people do on Wikipedia. It would be helpful to have more explicit things to onboard a research. There was some stuff of the structure of the data but more onboarding to explain how to use the coding schema.(P3)

Similarly, P3 noted that they did not understand the knowledge centered around the Barnstars coding example:

"Yeah, In some cases, especially for the Barnstars there is a lot of specific domain related knowledge that I didn't have related to what people do on Wikipedia." (P3)

The Barnstars coding example had our study participants code the comments that Wikipedia editors gave to justify giving an award to another editor. These editors sometimes use specific language related to Wikipedia behavior that may not be completely understood by readers who do not have experience in editing Wikipedia. Related to this issue of contextual familiarity, P5 noted that the language from the Alexa conversation task had language that was more clear and familiar to them:

"For the second task, I was more thorough going through because these are more straightforward language than acronyms. It helped me to go through and say yes/yes/no. If I did not know then I would go back and re-read. It was easier for me because in the Alexa one the language was more clear and it was helpful cause all of it was right there." (P5)

P19 gives an example from their own profession that demonstrates the need for contextualization of the coding schema. P19 discussed their own field site of oceanographers. In particular, they noted that oceanographers have different subdomains so it is important to understand how a person interprets and is connected to the data:

"I think having some other things like having a connection to the person who did the analysis, is something I want to see. But if the same person who collected the data is also doing the coding and the analysis and stuff, then obviously you are aware of all that stuff" (P19)

Another issue is information about how to apply a code. In most academic research papers, codes are explained but not necessarily with details about the process of applying the codes. Participants requested this additional metadata:

"The Alexa one I did not understand as well when you described it to me at first. I heard that you said that it was positive and negative examples but with each one having a yes/no or I felt like I had to make a call on all of them but then I realized that was not how it was. I think that is the most frequent error people make." (P2)

Similarly to P2, participants asked for more explicit instructions. One participant noted that the Alexa coding task needed additional information: 
"There were a couple of questions in the Alexa task because the definition felt like they had examples but I was not sure if this falls within the definition or not within the specific code. So some codes were a little bit more confusing. I wish there was more explicit instructions." (P3)

In our coding tasks, we did provide participants with instructions on how to complete the coding tasks external to the ontology. However, this participant requested additional instructions on how to apply the coding schema within the prototype.

Overall, participants felt that there was enough metadata to support the coding tasks but some of the current metadata was not needed for the coding tasks. P5 noted that consolidating some of the current information in the ontology might be more beneficial to their coding process:

"I felt like I was clicking through a bunch of things. There is an opportunity to consolidate the data. The bit I care about is to code so really interested in only the coding information."(P5)

\subsection{Switching strategies between the first and second coding task}

Switching between two unrelated coding tasks also presented some challenges for participants. In our prototype, the coding schemas were presented in two ways: 1) using a table with a list of the codes, and 2) a visualization to show the relationship between the codes. During the tasks, participants used different pieces of the system to understand what was happening. Additionally, the coding schemas included detailed descriptions and a lot of metadata. Moving back and forth between the coding schemas presented a challenge.

Most participants mentioned that their coding strategy was similar from the first task to the second. In the two coding tasks there were two different ways to apply the coding schema. The Barnstars coding schema had a hierarchical structure, where the participant first had to choose and overarching theme and then choose sub-codes that could fit under that theme. In the Alexa coding task, the participant had to first find if the anthropomorphic character existed and then had to choose for every code whether it was presented in a positive or negative way in the comment. These differences in reusing the coding schema led to minor changes in the strategy used by the participants to complete the coding task.

Some participants noted that it was easier to remember the Alexa coding scheme since it did not have a two layer structure. P7 completed the Barnstars coding task first. They noted their strategic changes helped handle the differences between coding schemas, especially since the Alexa coding task required not just the presence of a code but also if it was a positive or negative representation:

"A little bit, in the sense that the tasks were different. Here [Barnstars] I was just seeing if the code was present or not, in the second one [Alexa] I was seeing a positive or negative reaction. In the first one I was just trying to see if a code was present. In the second one, I was going by the category after having read the quote but in the other one I was going through them" (P7)

In constrast, P15 noted that the Alexa coding required much more processing since there were more codes and layer, that require more maneuvering back and 
forth from the prototype to the coding questions. P15 completed the Barnstars task first:

The first one [Barnstars] I think is really easy to remember all the codes and all the codes in the coding schema like two tasks. However, the second one [Alexa] I needed to go back to see the codes every time. Because it was it was more complex and it was super long. (P15)

P12 completed the Alexa task first and noted that the Barnstars coding task required more work since they had to work through 10 different codes for a layer of the coding task but for the first layer of Alexa, there was only 2 choices, positive or negative:

In the first coding schema [Alexa] there was nesting in the sense of you have to identify whether it affirms or negates, which is a very constrained type of coding scheme in a way. But it was one who did not require reference back to xx coding schema. If I identify that something is anthropormophized or making a statement about anthropomorphization, whether that is affirmative or negative is not a thing that I have to got often to check in the coding scheme. There is only two options in my head. If I determine something that is an edit, it could be any number of up to ten subcodes, so that is a thing where I was slower and I had to get back and take a look at them. (P12)

Their strategy was also highly dependent on how much knowledge they already had on the particular topic or research. P16, completed the Barnstars task first, mentioned that their strategy changed because they had better understanding of one coding task over the other:

Yeah, I think so. Maybe because. One of this had more codes and the other one was more straightforward and I think I could have coded it without necessarily having to read all the explanations in depth, but this one was more nuanced, I had to understand the difference between connectivity and skill. (P16)

P16 spent more time analyzing the codes for the Alexa coding task because they needed to understand context around the device. For the Barnstars task, it was not necessary to read all of the detailed explanations of codes but for the Alexa coding task it was needed to complete the task.

P17 completed the Barnstars task first and also noted that he needed to understand more about the research study in the Barnstars coding task which required him to look deeper into the research question for that particular coding task:

"I wanted to get a better understanding what the study was about and why what - So I guess when we are talking about social vs. collaborative, is this a paper that is looking at collaboration or is it more looking at complementing somebody or describing value to certain behavior or something. So specifically that required me to go beyond just the coding and actually go to the research question." (P17) 
7.6 Understanding how the ontology fits in a research workflow

After testing the prototype through two coding tasks, we asked the participants questions about problems they had with the ontology and QualiCO. The experimental environment of the ontology led to difficulties in the coding task. Participants reported obstacles completing both qualitative coding tasks using the ontology. There was no clear distinction between either task related to their preference for completing the tasks. 6 participants preferred Alexa, 5 preferred Barnstars and the remaining participants did not declare a preference.

One of the biggest challenges participants faced was understanding the purpose of the ontology. Most of the concerns about the system centered around the process of coding rather than actually reusing the coding schema. Participants faced tensions about whether the prototype/ontology are to help build/develop the coding process or whether it is a place (repository) for getting/reusing some of the code scheme. Participants were interested more in "how to do it now" and not necessarily the reuse part of the problem.

The prototype system we developed only allowed for a concrete representation of the final codes. Participants noted that creating a coding schema is a dynamic process and codes can shift. Currently, our ontology does not accommodate that need:

"Once you have a project that is mature, you have a stable coding schema but it takes a great deal of work to get up to that point and what we really have not seen or tested is the extent which it can support a living document that has this kind of history of codes that get subdivided or merged or codes that definitions had. (P10)

P11 also similarly asked for more detailed information that demonstrates the multiple stages of the coding process and the researchers involved. They note that the interpretation of a coding schema might be different and it is important for someone using a particular coding schema to understand its historical lineage.

Some of these have very lose or limited information about the development process. You know, sometimes it is just a couple of words. A lot of times it is helpful to get more detailed information about that. So it is not the kind of thing where you can force people to give extremely detailed information, but if I am being involved I do wanna know - you know - did they go through multiple stages of affinity diagramming? Did they - when they say they started with the data, where they borrowing anybody else's schema to begin with? You want a lot of rich content when you approach these things and there is only so much information you can present in a table like this. And you want your interpretations of the codes to be appropriately in line with what the original creators.. And if you find problems, another thing, I don't know if this is builtin, but a kind of feedback or comment process, if you find issues with the schema, like "I think it would be helpful to merge these or to add this other one. That could be helpful, if it is gonna be a collaborative process. (P11)

This quote from P11 shows that the participant is thinking of the prototype and ontology more as a tool for the coding process. Being able to integrate more of the 
coding process into the prototype would also be helpful for qualitative researchers to share their codes across a team. However, the QualiCO system was not developed to create coding schemas but rather to store coding schemas that had already been developed and used in prior researcher.

\section{Discussion}

In this section, we first answer the research questions, then we discuss the findings of our evaluation in detail and second what we learned from applying our method to an evaluation of the ontology. The section closes with the limitations of the research.

\subsection{Answering the research questions}

For the research questions, we can state the following:

RQ1: How does researcher performance change using the ontology across different coding schemas? Our evaluation showed that participants who had not worked with the coding schemas before were able to use the coding schemas in an assigned coding task. Improved time on task as well as improved accuracy suggest that QualiCO helped the participants to reuse the coding schemas in our coding task.

RQ2: Does a nearly complete instantiation of the ontology provide enough metadata for a researcher to reliably apply the coding schema? When we asked the participants whether information was missing, they only mentioned the usage of a code, more examples and versioning of the coding schemas. Apart from this, there were no issues mentioned. We conclude that a relatively full instantiation is sufficient to represent schema for potential reuse.

RQ3: How can this ontology be improved to make the reuse of coding schemas easier? We saw that many participants had issues with understanding special domain knowledge related to Wikipedia or Amazon's Alexa system. Participants were also interested in richer data about positive (application) and negative (omit) coding examples, and information about code frequency. We note that some of this information is commonly in publications that detail a qualitative coding research result.

Participants also mentioned that they were interested in understanding schema changes, potentially as part of the schema development, and tracking how schemas are reused. These are important aspects of qualitative schema sharing, but were outside the scope of our initial work to develop QualiCO. A future prototype that leveraged the QualiCO ontology could also versioning features like GIT, allowing researchers have a versioning structure and facilitating the possibility of forks when codding schemas are reused by other researchers. But we note these features would not require changes to the QualiCO ontology itself.

\subsection{Discussion of findings}

Next we reflect on our findings. We present some design recommendations and future directions for integrating these types of infrastructures into current approaches to fostering open science. Our results illustrate the potential for such platforms to give researchers new perspectives on coding schemas, better access to qualitative methods, better documentation for schema re-use, as well as potential support for team coding and improved traceability for qualitative research. 
Directly comparing the Alexa and Barnstar coding tasks is not really possible. They were adopted as our experimental tasks because we had access to previously coded data that had been consensus coded providing a highly reliable standard for the correct codes. The tasks had distinctly different structures that aligned with the underlying conceptual models of different participants. This is clear from the variety of preference statements we got from participants with no clear preference for one task over the other.

However, it is clear from our quantitative performance metrics that the Alexa task was "harder" in some sense. That is, regardless of whether the Alexa task was first or second, it took the participants more time and they were less accurate. We believe this is mostly a function of the constrained conditions of the experiment rather than a reflection of relative qualitative validity differences between the tasks.

Rather than comparing the specific tasks, we need to compare the experimental conditions. Looking at the time on task, we can see that the participants consistently performed faster in the second task, it did not matter whether this was the Barnstar or the Alexa task. The faster performance was not statistically significant. The task accuracy metric shows a similar trajectory. That is, our participants improved their coding accuracy regardless of which task was second. Again, overall, these accuracy improvements were not statistically significant. The combination of these two metrics, in the same positive direction, with the associated counter balanced control, is highly suggestive that the prototype and the ontology has some positive impact. If the prototype and QualiCO ontology were too complex, one or both of these metrics might be otherwise oriented.

Our qualitative interview data revealed a number of interesting aspects with regard to the specifics of the tasks, aspects of the QualiCO ontology, and, perhaps most interestingly, how the participants conceptualized the prototype.

One issue with the experimental tasks was that both of them needed some domain specific knowledge to be performed well. Differences in this domain knowledge might explain why some participants preferred one task over the other. This shows that our instantiation of the coding schemas had some metadata gaps. For example, including metadata on specific publications that used the schema and metadata on the research data, sources and context, would be helpful. This also illustrates a specific challenge when using a task-based evaluation of an ontology system. The participants familiarity with the task domain influences the outcomes for the ontology system.

Embedded in some of the quotes from our participants are underlying assumptions about what our prototype tool was designed to achieve. That is, despite our efforts to focus the participants on the underlying ontology, what it represented and what it contained, participants still answered questions by "framing" their answers in their experiences of prior qualitative coding work similar to the phenomena of technological perceptions described in XX. Many participants framed the platform as a tool that they could use for conducting qualitative research rather than for sharing qualitative schemas or finding an open schema on which to build.

One frame was to think of our prototype as a tool for conducting their qualitative coding activity. Some of our participants had used commercial QDA-software before, while others only had experience coding through spreadsheets. We made an 
explicit attempt to discourage this framing by having the participants specifically code on paper based coding sheets. With this frame, the prototype and the ontology provided access to the schema and some examples, but did not provide a way to apply codes to data. And, during interviews participants noted that they probably would not use the tool for conducting their coding. This does not actually illustrate a flaw with the QualiCO ontology. It does illustrate some conceptual barriers for incorporating open science practice among research disciplines that leverage qualitative methods.

A similar framing of the tool is as a collaboration platform. This framing is closer to how the prototype was explained. Finding a shared schema or sharing a schema is a type of collaboration. That the participants would see collaboration as present in the prototype seems understandable. However, this was still framed through the participants' experiences of practice. Several, thought of the prototype as a potential tool for collaboratively developing a schema. This is evident in interview answers where the participants mention refinement or versioning of the individual codes. Some participants were framing the tool as a mechanism for tracking the work of developing the schema. This framing has a potential positive side effect. If a tool facilitated the development of the schema, then there would be little effort necessary to share that final version. The prototype we tested explained a model where researchers upload and publish their schema after the schema and associated research was completed. Participants who framed our prototype as a collaboration tool saw distinct benefit in having an elaborated description of a schema in a place where other members of a collaborative research effort could find it.

We saw another important conceptual framing of the prototype that may not be obvious. The experimental tasks were specifically a methods task; applying codes to data. However, we noticed that some participants thought the prototype was more about sharing data than the sharing of method. The rhetoric and practices around open science has been focused on data sharing as that is dominant in much of the prior literature. The participants in our study are at least tacitly aware of this. Several participants stuck to this "open science is data sharing" frame of reference even when we asked them about sharing methods. This is not explicitly a flaw in QualiCO nor our research design. This does illustrate that among qualitative research practitioners that constituents of open science are shaped and framed by the popular or dominate conversations.

For research in open science, our study shows that reuse of coding schemas is possible given reasonably rich metadata for the schemas. QualiCO bridges an important gap between pure archiving of coding schemas and allowing for the sharing of methods within qualitative research. Professional science societies have called to fill this gap (e.g., APA guidelines [34]) through data and method sharing that aligns with the information researchers would need to provide QualiCO. QualiCO could help address the calls of these professional societies. Still, documenting qualitative practices and creating incentive structures to publicly share the details of methods and schemas will require significant community effort [25].

\subsection{Usability testing Ontologies}

In Section 4.3 we described several approaches to evaluating ontologies. We conducted a task-based evaluation that focused the participants on a methods reuse 
problem. Given the range of methods that fall under the umbrella of qualitative methods, our approach might not be the only strategy to test an ontology. However, prior work shows that it is hard to test an ontology to the exclusion of a system. Our specific approach attempted to focus participants on the QualiCO ontology and background the prototype as much as possible. Based on the interviews, we believe that our approach worked. However, as we point out above, there can still be conceptual issues that influence the participants responses and that shift the focus of the participant back on a prototype software. Part of the success may have been the choice of using a wiki (i.e., Semantic MediaWiki) for the prototype implementation. In general, the wiki provides a minimal interface and the QualiCO ontology was therefore rather explicit in the linking structure that connected the metadata for the tasks.

We claim that ontologies like QualiCO can and should be tested against realworld tasks, especially when they describe data or methods that are meant to be reused. However, we recognize that there are important methodological issues in these evaluations that are not simple to resolve. For example, our specific experimental design put QualiCO at a disadvantage in comparison to real-world coding practices. Given the time limits, participants were not able to read prior publications, talk to the researchers who developed the coding schema or follow up on task domain knowledge that might have been helpful. We had participants explicitly mention these challenges. Still, our participants were able to complete the assigned tasks, suggesting that the metadata supplied was at least minimally sufficient, and demonstrating that a real-world task-based evaluation is reasonable.

\subsection{Limitations}

We note that there are several limitations to this research. First, we have focused on one particular qualitative research method. We focused on systematic qualitative coding because it occupies a philosophical space of research that is relatively close to the positivist stance of most research methods that have been the focus of the majority of prior research in open science. Evaluating whether QualiCO can address other methods in the qualitative tradition is open for future research.

As in many experiments, the experimental conditions do not exactly mirror the real-world conditions in which this type of qualitative coding activity would happen. However, we believe that our experimentation conditions create a type of deficit for the ontology that is not explained away through experimental demand characteristics nor simple learning. While we do not have statistical significance in the experiment, we believe the direction of the implications point to some benefits derived from QualiCO.

Our evaluation relied on one specific prototype that represented the ontology in one specific way. One can imagine that other prototyping techniques or a fullblown application might reflect the QualiCO ontology in a slightly different way. Focusing on just one prototype was important to begin a systematic evaluation. Future evaluations might approach the implementation of QualiCO in a prototype differently.

Another specific limitation is how we instantiated the ontology to reflect the two experimental conditions. In this work we specifically biased toward providing rich 
metadata for the schemas. We filled as many of the QualiCO fields and relations as possible. This reflects a particular baseline. An alternative experiment could vary the amount of metadata for each experimental schema. For example, it might have been interesting to see at what minimum of metadata the prototype becomes unusable. However, we believe that would not be helpful as a first evaluation to illustrate basic utility of the ontology and prototype. Our focus was to evaluate the ontology itself and not some range of instantiations of the ontology.

Lastly, our participants largely came from one disciplinary perspective - Human Centered Design. While we solicited in several different academic and research units, we were not able to garner broad participation across those units. We believe that systematic qualitative coding is largely taught and practiced in similar ways across a number of disciplines, but it is possible that there are factors among the set of participants that generated our results.

\section{Conclusion}

In this evaluation we tested the ontology QualiCO. Our goal was to understand whether QualiCO could be used to understand a previously unknown systematic qualitative coding schema. We tested this by creating two coding tasks and having participants attempt to code sample data using schemas entered into a prototype system.

QualiCO was designed to bridge the gap between simply archiving coding schemas to sharing of supplemental information to research, like research data. With our prototype implementation, we show the potential for method sharing in qualitative research using QualiCO. A description of a coding schema using QualiCO enables researchers to share their coding schemas and therefore also their methods.

The next steps will be the implementation of QualiCO within research data centers. The complete ontology can be found on GitHub ${ }^{[6]}$, anyone interested in implementing the ontology is welcome to get in touch with us.

\section{Appendix}

\section{Acknowledgements}

The authors would like to thank Mark Rittberger, Christoph Schindler, Christa Womser-Hacker and Thomas Mandl for providing feedback throughout the whole research project.

Funding

Not applicable.

Availability of data and materials

The datasets supporting the conclusions of this article are available in the Zenodo repository,

https://doi.org/10.5281/zenodo.4442523. In the repository you can find all raw data about the performance on the tasks, including the calculation on how we rated the tasks as well as t-tests and time on task. In the repository, you can also find the system comprehension quiz, task description and interview guidelines we used to conduct the tests.

Ethics approval and consent to participate

Not applicable.

Competing interests

The authors declare that they have no competing interests.

Consent for publication

Not applicable.

Authors' contributions

Not applicable.

\footnotetext{
${ }^{[6]}$ https://github.com/julianhocker/Quali-Codes-Ontology
} 
Authors' information

Not applicable.

\section{Author details}

${ }^{1}$ DIPF - Leibniz Institute for Research and Information in Education, Frankfurt am Main, Germany.

${ }^{2}$ Human-centered design and endineering, University of Washington, Washington, US.

\section{References}

1. Google: Google Books Ngram Viewer (2020).

https: //books . google. com/ngrams/graph? content=open+science\&case_insensitive=on\&year_start= $1800 \&$ year_end $=2008 \&$ corpus $=15 \&$ smoothing $=3 \&$ share $=\&$ direct_ur $1=t 4 \% 3 \mathrm{~B} \% 2 \mathrm{Copen} \% 20$ science $\% 3 \mathrm{~B} \% 2 \mathrm{Cc0} \% 3 \mathrm{~B} \%$ $2 \mathrm{Cs} 0 \% 3 \mathrm{~B} \% 3 \mathrm{~B}$ open $\% 20$ science $\% 3 \mathrm{~B} \% 2 \mathrm{Cc0} \% 3 \mathrm{~B} \% 3 \mathrm{BOpen} \% 20$ Science $\% 3 \mathrm{~B} \% 2 \mathrm{Cc0} \% 3 \mathrm{~B} \% 3 \mathrm{~B}$ open $\% 2$ Science $\% 3 \mathrm{~B} \% 2 \mathrm{Cc0} \% 3 \mathrm{~B} \%$

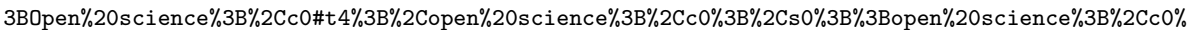
$3 \mathrm{~B} \% 3 \mathrm{BOpen} \% 20$ Science $\% 3 \mathrm{~B} \% 2 \mathrm{Cc0} \% 3 \mathrm{~B} \% 3 \mathrm{~B}$ open $\% 20$ Science $\% 3 \mathrm{~B} \% 2 \mathrm{Cc} 0 \% 3 \mathrm{~B} \% 3 \mathrm{BOpen} \% 20$ science $\% 3 \mathrm{~B} \% 2 \mathrm{Cc0}$

2. Collaboration, O.S., et al.: Estimating the reproducibility of psychological science. Science 349(6251), 4716 (2015)

3. Fischer, B.A., Zigmond, M.J.: The essential nature of sharing in science. Science and engineering ethics 16(4), 783-799 (2010)

4. Louis, K.S., Jones, L.M., Campbell, E.G.: Macroscope: sharing in science. American Scientist 90(4), 304-307 (2002)

5. Madill, A.: Qualitative research is not a paradigm: Commentary on jackson (2015) and landrum and garza (2015). (2015)

6. DuBois, J.M., Strait, M., Walsh, H.: Is it time to share qualitative research data? Qualitative Psychology 5(3), $380(2018)$

7. Bücker, N.: How to code your qualitative data-a comparison between grounded theory methodology and qualitative content analysis. In: Forum Qualitative Sozialforschung/Forum: Qualitative Social Research, vol. 21 (2020)

8. Hocker, J., Schindler, C., Rittberger, M.: Participatory design for ontologies. a case study of an open science ontology for qualitative coding schemas. Aslib Journal of Information Management (2020). in print

9. Huang, X., Hawkins, B.A., Lei, F., Miller, G.L., Favret, C., Zhang, R., Qiao, G.: Willing or unwilling to share primary biodiversity data: results and implications of an international survey. Conservation Letters 5(5), 399-406 (2012). doi:10.1111/j.1755-263X.2012.00259.x. https://conbio.onlinelibrary.wiley.com/doi/pdf/10.1111/j.1755-263X.2012.00259.x

10. Melero, R., Navarro-Molina, C.: Researchers' attitudes and perceptions towards data sharing and data reuse in the field of food science and technology. Learned Publishing 33(2), 163-179 (2020). doi:10.1002/leap.1287. https://onlinelibrary.wiley.com/doi/pdf/10.1002/leap.1287

11. Kim, Y., Nah, S.: Internet researchers' data sharing behaviors. Online Information Review (2018)

12. Tenopir, C., Christian, L., Allard, S., Borycz, J.: Research data sharing: Practices and attitudes of geophysicists. Earth and Space Science 5(12), 891-902 (2018). doi:10.1029/2018EA000461. https://agupubs.onlinelibrary.wiley.com/doi/pdf/10.1029/2018EA000461

13. Birnholtz, J.P., Bietz, M.J.: Data at work: supporting sharing in science and engineering. In: Proceedings of the 2003 International ACM SIGGROUP Conference on Supporting Group Work, pp. 339-348 (2003)

14. Rodrigues, J., Castro, J.A., da Silva, J.R., Ribeiro, C.: Hands-on data publishing with researchers: Five experiments with metadata in multiple domains. In: Italian Research Conference on Digital Libraries, pp. 274-288 (2019). Springer

15. Wilkinson, M.D., Dumontier, M., Aalbersberg, I.J., Appleton, G., Axton, M., Baak, A., Blomberg, N., Boiten, J.-W., da Silva Santos, L.B., Bourne, P.E., et al.: The fair guiding principles for scientific data management and stewardship. Scientific data 3(1), 1-9 (2016)

16. Alliance, G.I.D.: Care principles for indigenous data governance. GIDA https://www. gida-global. org/care (2019)

17. Kowalczyk, S., Shankar, K.: Data sharing in the sciences. Annual review of information science and technology 45(1), 247-294 (2011)

18. Faniel, I.M., Kriesberg, A., Yakel, E.: Social scientists' satisfaction with data reuse. Journal of the Association for Information Science and Technology 67(6), 1404-1416 (2016)

19. Curty, R.G.: Factors influencing research data reuse in the social sciences: An exploratory study. International Journal of Digital Curation (2016). doi:10.2218/ijdc.v11i1.401

20. Studer, R., Benjamins, V.R., Fensel, D.: Knowledge engineering: principles and methods. Data \& knowledge engineering 25(1-2), 161-197 (1998)

21. Borgman, C.L.: Big Data, Little Data, No Data: Scholarship in the Networked World. MIT press, ??? (2015)

22. Heidorn, P.B.: Shedding light on the dark data in the long tail of science. Library trends 57(2), 280-299 (2008)

23. Mays, N., Pope, C.: Quality in Qualitative Research, pp. 211-233. John Wiley \& Sons, Ltd, ??? (2020). Chap. 15. https://onlinelibrary.wiley.com/doi/pdf/10.1002/9781119410867.ch15. doi:10.1002/9781119410867.ch15. https://onlinelibrary.wiley.com/doi/abs/10.1002/9781119410867.ch15

24. LONG, A.F., GODFREY, M.: An evaluation tool to assess the quality of qualitative research studies. International Journal of Social Research Methodology 7(2), 181-196 (2004). doi:10.1080/1364557032000045302. https://doi.org/10.1080/1364557032000045302

25. Hocker, J., Bipat, T., Zachry, M., McDonald, D.W.: Sharing your coding schemas: Developing a platform to fit within the qualitative research workflow. In: Proceedings of the 16th International Symposium on Open Collaboration, pp. 1-10 (2020)

26. Glaser, B.G., Strauss, A.L.: Discovery of Grounded Theory: Strategies for Qualitative Research. Routledge, ??? (2017)

27. Saldaña, J.: The Coding Manual for Qualitative Researchers. Sage, ??? (2015) 
28. Mayring, P., Brunner, E.: Qualitative inhaltsanalyse. In: Friebertshäuser, B., Langer, A., Prengel, A. (eds.) Handbuch Qualitative Forschungsmethoden in der Erziehungswissenschaft, pp. 323-334. Juventa-Verl.,, Weinheim ; (2013). http://swbplus.bsz-bw.de/bsz308206126inh.htm

29. Schreier, M.: Qualitative Content Analysis in Practice. Sage Publications, ??? (2012)

30. Scheliga, K., Friesike, S.: Putting open science into practice: A social dilemma? First Monday 19(9) (2014). doi:10.5210/fm.v19i9.5381

31. Aguinis, H., Solarino, A.M.: Transparency and replicability in qualitative research: The case of interviews with elite informants. Strategic Management Journal 40(8), 1291-1315 (2019). doi:10.1002/smj.3015. https://onlinelibrary.wiley.com/doi/pdf/10.1002/smj.3015

32. Grubb, A.M., Easterbrook, S.M.: On the lack of consensus over the meaning of openness: An empirical study. PLOS ONE 6(8), 1-12 (2011). doi:10.1371/journal.pone.0023420

33. Kapiszewski, D., Karcher, S.: Transparency in practice in qualitative research. APSA Preprints (2019). doi:10.33774/apsa-2019-if2he-v2

34. Levitt, H.M., Bamberg, M., Creswell, J.W., Frost, D.M., Josselson, R., Suárez-Orozco, C.: Journal article reporting standards for qualitative primary, qualitative meta-analytic, and mixed methods research in psychology: The apa publications and communications board task force report. American Psychologist 73(1), 26 (2018)

35. Evers, J., Caprioli, M.U., Nöst, S., Wiedemann, G.: What is the refi-qda standard: Experimenting with the transfer of analyzed research projects between qda software. In: Forum Qualitative Sozialforschung/Forum: Qualitative Social Research, vol. 21 (2020)

36. Strauss, A., Corbin, J.: Grounded theory methodology. Handbook of qualitative research 17, $273-85$ (1994)

37. Auer, S., Kovtun, V., Prinz, M., Kasprzik, A., Stocker, M., Vidal, M.E.: Towards a knowledge graph for science. In: Proceedings of the 8th International Conference on Web Intelligence, Mining and Semantics, pp. 1-6 (2018)

38. McDaniel, M., Storey, V.C.: Evaluating domain ontologies: Clarification, classification, and challenges. ACM Comput. Surv. 52(4) (2019). doi:10.1145/3329124

39. Palavitsinis, N.: Metadata quality issues in learning repositories (2014)

40. $\mathrm{Hu}, \mathrm{X} ., \mathrm{Ng}, \mathrm{J} ., \mathrm{Xia}, \mathrm{S}$.: User-centered evaluation of metadata schema for nonmovable cultural heritage: Murals and stone cave temples. Journal of the Association for Information Science and Technology 69(12), 1476-1487 (2018). Online verfügbar unter: https://doi.org/10.1002/asi.24065

41. Lee, J.H., Clarke, R.I., Perti, A.: Empirical evaluation of metadata for video games and interactive media. Journal of the Association for Information Science and Technology 66(12), 2609-2625 (2015). doi:10.1002/asi.23357. https://asistdl.onlinelibrary.wiley.com/doi/pdf/10.1002/asi.23357

42. Malone, J., Parkinson, H.: Reference and Application Ontologies. http://ontogenesis.knowledgeblog.org/295 (2010). http://ontogenesis.knowledgeblog.org/295

43. Reinhold, A.: Forschungsdaten in der Videobasierten Unterrichtsforschung: Benutzerzentrierte Modellierung und Evaluierung Einer Domänen-Ontologie. Hülsbusch, ??? (2015)

44. Liu, D., Bikakis, A., Vlachidis, A.: Evaluation of semantic web ontologies for modelling art collections. In: Kirikova, M., Nørvåg, K., Papadopoulos, G.A., Gamper, J., Wrembel, R., Darmont, J., Rizzi, S. (eds.) New Trends in Databases and Information Systems, pp. 343-352. Springer, Cham (2017)

45. Yu, J., Thom, J.A., Tam, A.: Requirements-oriented methodology for evaluating ontologies. Information Systems 34(8), 766-791 (2009). doi:10.1016/j.is.2009.04.002. Sixteenth ACM Conference on Information Knowledge and Management (CIKM 2007)

46. Pittet, P., Barthélémy, J.: Exploiting Users' Feedbacks: Towards a Task-based Evaluation of Application Ontologies throughout Their Lifecycle. In: International Conference on Knowledge Engineering and Ontology Development. Proceedings of the International Conference on Knowledge Engineering and Ontology Development, part of the 7th International, Joint Conference on Knowledge Discovery, Knowledge Engineering and Knowledge Management (IC3K 2015), vol. 2. Lisbonne, Portugal (2015). https://hal.archives-ouvertes.fr/hal-01459827

47. Kriplean, T., Beschastnikh, I., McDonald, D.W.: Articulations of wikiwork: Uncovering valued work in wikipedia through barnstars. In: Proceedings of the 2008 ACM Conference on Computer Supported Cooperative Work. CSCW '08, pp. 47-56. Association for Computing Machinery, New York, NY, USA (2008). doi:10.1145/1460563.1460573. https://doi.org/10.1145/1460563.1460573

48. McDonald, D.W., Javanmardi, S., Zachry, M.: Finding patterns in behavioral observations by automatically labeling forms of wikiwork in barnstars. In: Proceedings of the 7th International Symposium on Wikis and Open Collaboration. WikiSym '11, pp. 15-24. Association for Computing Machinery, New York, NY, USA (2011). doi:10.1145/2038558.2038562. https://doi.org/10.1145/2038558.2038562

49. Friedman, B., Kahn Jr, P.H., Hagman, J.: Hardware companions? what online aibo discussion forums reveal about the human-robotic relationship. In: Proceedings of the SIGCHI Conference on Human Factors in Computing Systems, pp. 273-280 (2003)

\section{Additional Files}

Not applicable. 
Figures

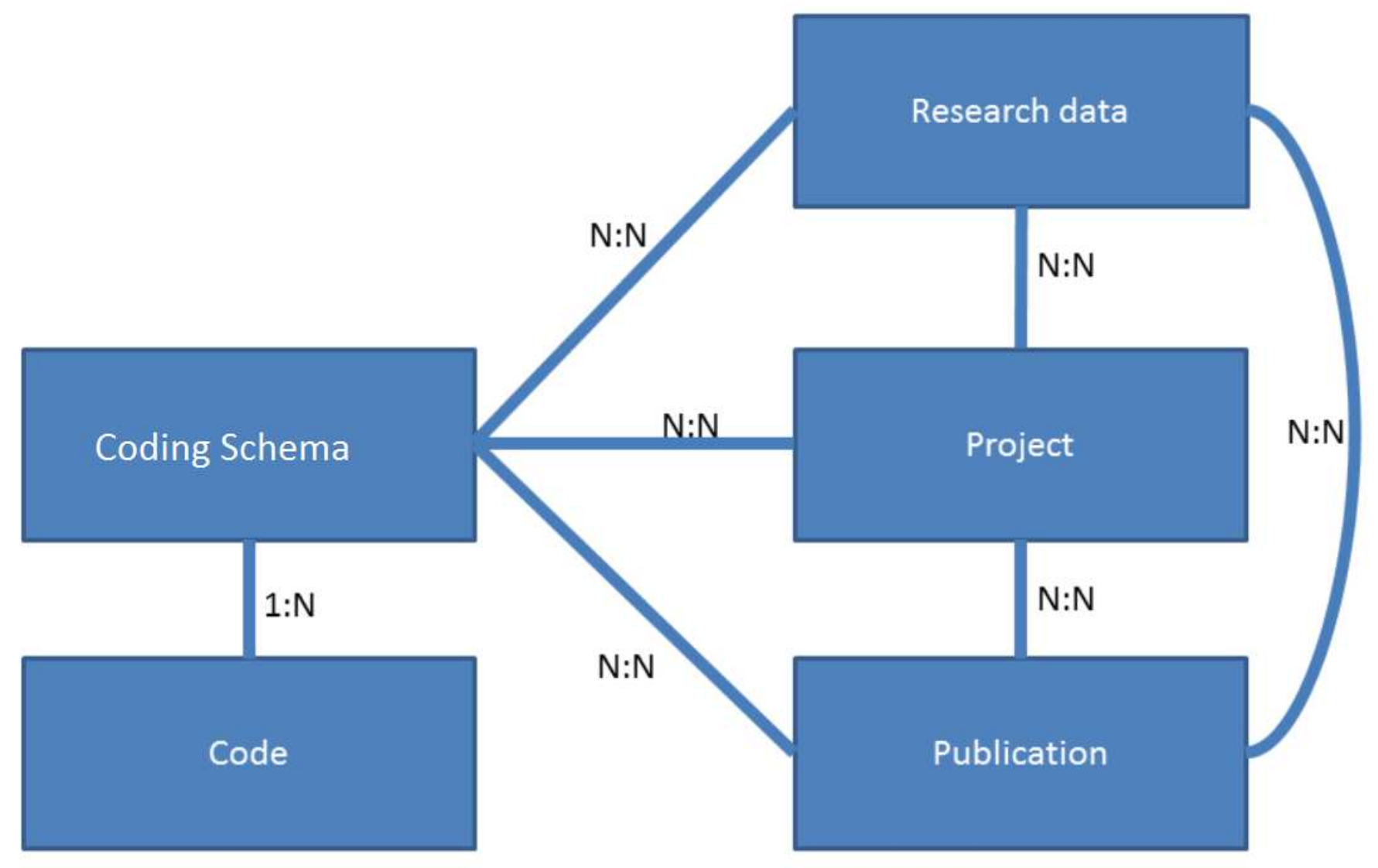

Figure 1

Structure of the ontology. 


\section{Barnstars - coding schema}

\begin{tabular}{|c|c|}
\hline Author & David W. McDonald \\
\hline Research questions & How are Barnstars used in Wikipedia? How are people awarded with Barnstars? \\
\hline Method & Qualitative codin \\
\hline Theoretical background & $\begin{array}{l}\text { The codebook contains three groups of codes: (1) the scope } \\
\text { of the barnstar, such as whether it points to a specific edit or comments on general contributions to Wikipedia, (2) the genre of the barnstar, } \\
\text { such as whether it is antagonistic, and (3) the work that the barnstar acknowledges. }\end{array}$ \\
\hline Process of creation & $\begin{array}{l}\text { codebook by open coding a random sample of } 200 \text { barnstars. } \\
\text { We then applied the codebook to a second random sample of } 200 \text { barnstars. Through our attempt to systematically code the second barnstar } \\
\text { sample, we iteratively refined our codes and agreed upon their application to the barnstars. }\end{array}$ \\
\hline $\begin{array}{l}\text { Dimensionalization and } \\
\text { coding cycles }\end{array}$ & $\begin{array}{l}\text { We used this codebook to iteratively code a random sample of } 2400 \text { barnstars. The barnstars were divided randomly into six bins. Two of the } \\
\text { authors independently coded each bin. One coder reviewed the codes and noted discrepencies. Discrepencies were iterated upon until there } \\
\text { was consensus. After coding was completed, we performed extensive consistency checking of commonly correlated codes. } \\
\text { A barnstar often suggests multiple legitimate codings, either because a particular phrasing calls out multiple dimensions or because the } \\
\text { barnstar contains multiple independent statements. We therefore chose to apply multiple codes for every code group instead of force-fitting a } \\
\text { dominant code. For example, the following barnstar provides evidence of a prior encounter and serves as a peace offering (genre), while } \\
\text { acknowledging general administratative actions, committment to the project, and conflict mediation (work) }\end{array}$ \\
\hline Date & 2008 \\
\hline Research area & Information Science \\
\hline Inter-coder-reliability & $85 \%$ \\
\hline Keywords & Wikipedia, CSCW \\
\hline \multicolumn{2}{|l|}{ Visualizations } \\
\hline Software & NVivo \\
\hline Coding schema & Datei:Codes.xml \\
\hline Project data & Datei:Codes.xml \\
\hline Research data & Barnstars - research data \\
\hline Publication & Articulations of WikiWork, Finding Patterns in Behavioral Observations by Automatically Labeling Forms of Wikiwork in Barnstars \\
\hline Study & Barnstar Study \\
\hline
\end{tabular}

\section{Figure 2}

Overview of metadata for codding schema for the Barnstars task. 


\begin{tabular}{|c|c|c|c|}
\hline$\bullet$ & Definition & Example & $\begin{array}{c}\text { Has } \\
\text { Successor }\end{array}$ \\
\hline $\begin{array}{l}\text { Collaborative } \\
\text { Action }\end{array}$ & $\begin{array}{l}\text { Collaborative Actions and Disposition is differentiated from Social } \\
\text { and Community Support Actions by the direct implication of } \\
\text { collaborative activity, such as conflict mediation on talk pages. } \\
\text { Although this category points to work activities that involve others, } \\
\text { it is important to recognize that the dependencies are not clearly } \\
\text { present in the texts of the barnstars }\end{array}$ & $\begin{array}{l}\text { It is my honor to award Anonymous Editor this...green } \\
\text { cucumber with sunglasses, for being cool when the } \\
\text { editing isn't. For always keeping a level head. If you } \\
\text { can keep your head when all around you are losing } \\
\text { theirs and blaming it on you }\end{array}$ & Root \\
\hline Meta Content & $\begin{array}{l}\text { The last category is work related to meta-content. Meta-content } \\
\text { work includes the acknowledgement of tool creation } \\
\text { (programming), creation of templates, creation and management } \\
\text { of categories or category tags, and work on formal Wikipedia } \\
\text { policies. }\end{array}$ & $\begin{array}{l}\text { I award you this fine barnstar for the work you did in } \\
\text { improving the Taxobox template by adding } \\
\text { conservation status shortcuts. I was thinking of doing } \\
\text { that myself, but you beat me to the punch! }\end{array}$ & Root \\
\hline Social/Community & $\begin{array}{l}\text { This category includes welcoming newcomers, initiating or leading } \\
\text { new projects, rewarding individuals who give out barnstars, and } \\
\text { general social support. In the examples below we see individuals } \\
\text { receiving barnstars for their personal characteristics and } \\
\text { willingness to help other Wikipedians }\end{array}$ & $\begin{array}{l}\text { I hereby ordain Andhrimnir to "The Order of the } \\
\text { Smiley", for his dedication in helping newcomers like } \\
\text { me. Without people like you, we would be lost. } \\
\text { Awarded by Hermod }\end{array}$ & Root \\
\hline Administrative & $\begin{array}{l}\text { The barnstars in this category pertain to the actions taken by } \\
\text { administrators and acknowledge participation in formal processes. } \\
\text { Common processes include Editor Reviews and Featured Article } \\
\text { Reviews }\end{array}$ & $\begin{array}{l}\text { I hereby award you The Working Man's Barnstar for } \\
\text { repeatedly notifying relevant parties during featured } \\
\text { article reviews, as it's an oft-forgotten task. }\end{array}$ & Root \\
\hline Editing & $\begin{array}{l}\text { Acknowledgments for copy editing, general editing, and for } \\
\text { contributions to specific articles }\end{array}$ & $\begin{array}{l}\text { Mani, you have contributed a great deal of Estonian } \\
\text { articles and done major and useful copyedits in a } \\
\text { short time. You are a very productive user and } \\
\text { deserve recognition. I award you, Magni, this } \\
\text { Barnstar for keeping on top of this Wikipedia article } \\
\text { and for being the "Master Editor". Keep up the fine } \\
\text { work! }\end{array}$ & Root \\
\hline Misc/Unknown & $\begin{array}{l}\text { There are some barnstars that are not very specific about the } \\
\text { actual work completed. Many such barnstars include references } \\
\text { to "Janitorial Services" and "mop and bucket", reflecting a general } \\
\text { work ethic of cleaning and maintaining various aspects of } \\
\text { Wikipedia. }\end{array}$ & $\begin{array}{l}\text { I, Gerd, award you this barnstar for your hard work } \\
\text { here at Wikipedia, and to let you know I feel your wiki- } \\
\text { stress. Gefion awards this Barnstar to Frigg for } \\
\text { dedicated hard work with the mop and bucket, } \\
\text { making Wikipedia better for everyone. }\end{array}$ & Root \\
\hline Border Patrol & $\begin{array}{l}\text { Detecting and disciplining such behavior constitutes a wide range } \\
\text { of punitive work in Wikipedia. The most acknowledged border } \\
\text { patrol activity is fighting vandalism by reverting or repairing the } \\
\text { damage. }\end{array}$ & $\begin{array}{l}\text { For standing with me this morning to revert all of } \\
\text { those } A O L \text { vandals hell-bent on causing as much } \\
\text { trouble as possible, I award you this barnstar. }\end{array}$ & Root \\
\hline
\end{tabular}

\section{Figure 3}

Table representation of top-level codes in the task Barnstars. 


\section{First Level of coding [Bearbeiten]}

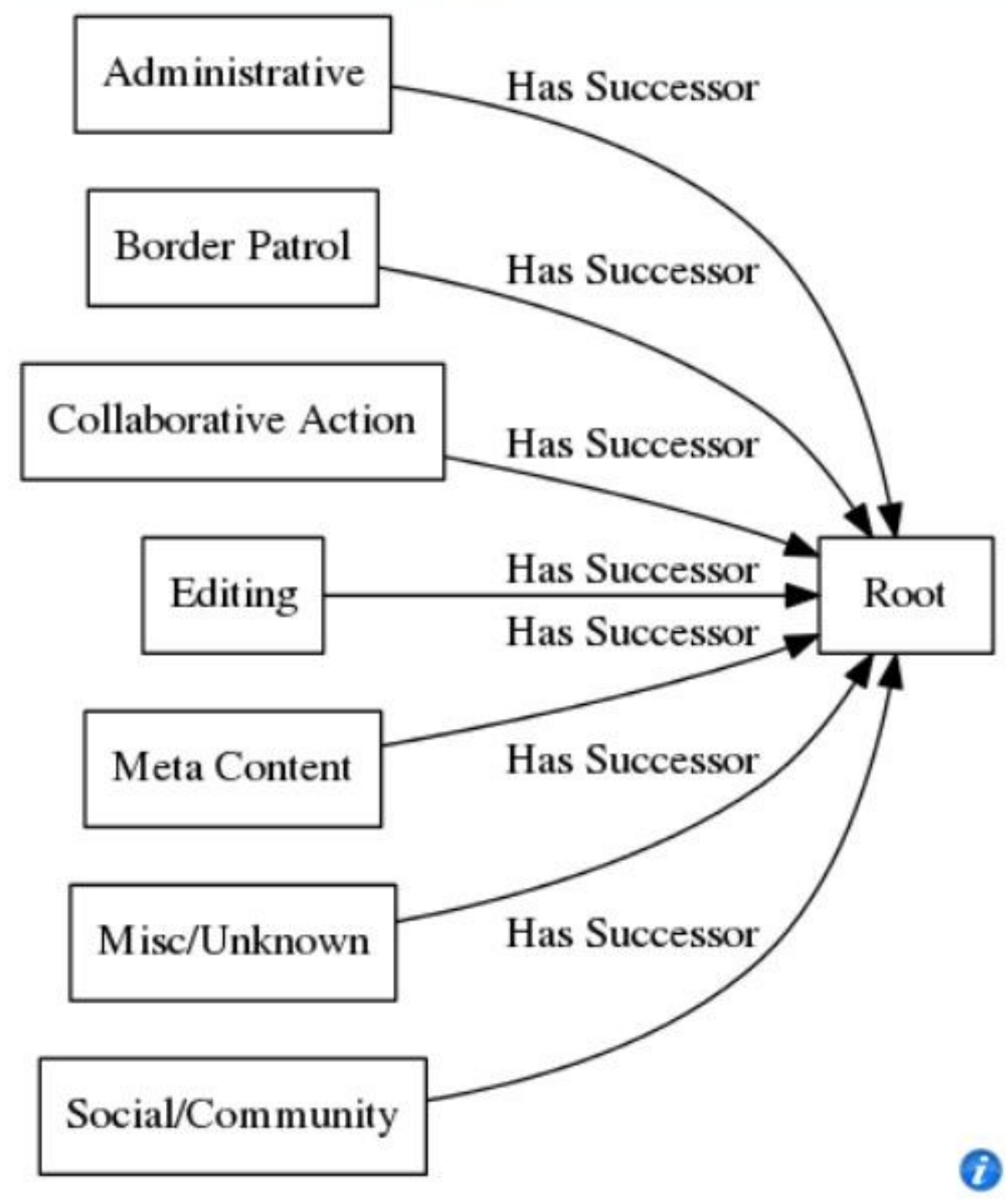

Figure 4

Graphical representation of top-level codes in the task Barnstars. 
article editing

\begin{tabular}{|l|l|}
\hline EG & general editing \\
\hline EMAJ & contributions-major (MAJOR CHANGES TO TEXT; some kind of large refinement or improvement to the article) \\
\hline EMED & contributions-media (images, audio, graphic design work, photos, latex) \\
\hline EINI & initiative (starting an article, work on article stubs) \\
\hline ERED & redesign / restructuring (refactoring, merging pages) \\
\hline EMIN & minor (cleanup, copy-editing, maintenance, work on captions, work on a single article) \\
\hline EACH & achievement (FA, FP, GA, DYK, readable) \\
\hline ECLA & classification (categorization, disambiguating links, redirects) \\
\hline EATT & attribution (adding references; removing unattributable information) \\
\hline ETRA & translation (to another language) \\
\hline EEXT & external research (doing outside research in order to make quality contributions) \\
\hline
\end{tabular}

collaborative actions/disposition (individual attribute)

\begin{tabular}{|l|l|}
\hline CG & general collaborative \\
\hline CDA & diplomatic-action (working toward concensus, mediation) \\
\hline CDD & diplomatic-disposition (staying cool, civility, maintaining sense of humor, accepting of criticism, cooperative/collaborative) \\
\hline CEXP & explanation (instance of explanation; to newcomers, of particular area of expertise, intricacy of policy) \\
\hline CADH & adherence (to policy, maintaining NPOV, balancing policies, academic integrity) \\
\hline
\end{tabular}

meta content

\begin{tabular}{|l|l|}
\hline MG & general meta \\
\hline MPRO & programming artifacts (external tools, bots) \\
\hline MCLA & classification (category creation, refactoring) \\
\hline MPOL & policy / formal process (creation, refactoring, elaboration) \\
\hline MTEM & template (creation, refactoring, barnstars, page templates, userboxes, DYK) \\
\hline MFOR & forums / portals (creation, maintenance) \\
\hline MARC & archiving (user talk pages, discussion pages) \\
\hline
\end{tabular}

\section{Figure 5}

Detailed description of codes in the Barnstars task. 


\begin{tabular}{|c|c|}
\hline Author & Taryn Bipat \\
\hline Research questions & How do consumer's use the echo? Were expectations of using the Echo met? What terminology do consumers use to address the Alexa and the Echo? \\
\hline Method & Coding of the data with pre-defined and refined coding schema \\
\hline Theoretical background & [[Theoretischer Hintergrund::Coding Schema based on Coding schema for Hardware Companions; Value Sensitive Design (VSD) methods.]] \\
\hline Process of creation & $\begin{array}{l}\text { A code book was produced using the pilot data. This code book included explanations on how to code this corpus of data and the characteristics of each code that } \\
\text { could be found in the qualitative data After the generation of the code book, the systematic codes were used to analyze the remainder of the verified product } \\
\text { reviews. The coding was fixed in order to get correct percentages if a user used the same code multiple times. Many of the posts included more than one code } \\
\text { and were coded accordingly so that each code was only counted once per participant. }\end{array}$ \\
\hline $\begin{array}{l}\text { Dimensionalization and } \\
\text { coding cycles }\end{array}$ & [[Dimensionalisierung::The codes from Coding schema for Hardware Companions was adopted to the needs of the study.]] \\
\hline Date & 2018 \\
\hline Research area & Information Science \\
\hline Inter-coder-reliability & $85 \%$ \\
\hline \multicolumn{2}{|l|}{ Keywords } \\
\hline \multicolumn{2}{|l|}{ Visualizations } \\
\hline Software & Atlas TI \\
\hline Coding schema & Datei:Codes.xml \\
\hline Project data & Datei:Codes $\mathrm{xml}$ \\
\hline Research data & Research data for Voice Conversational Agents \\
\hline Publication & Voice Conversational Agents: A Value Sensitive Approach \\
\hline Study & Voice conversational agents - study \\
\hline
\end{tabular}

\section{Figure 6}

Overview of metadata for the Alexa coding schema. 


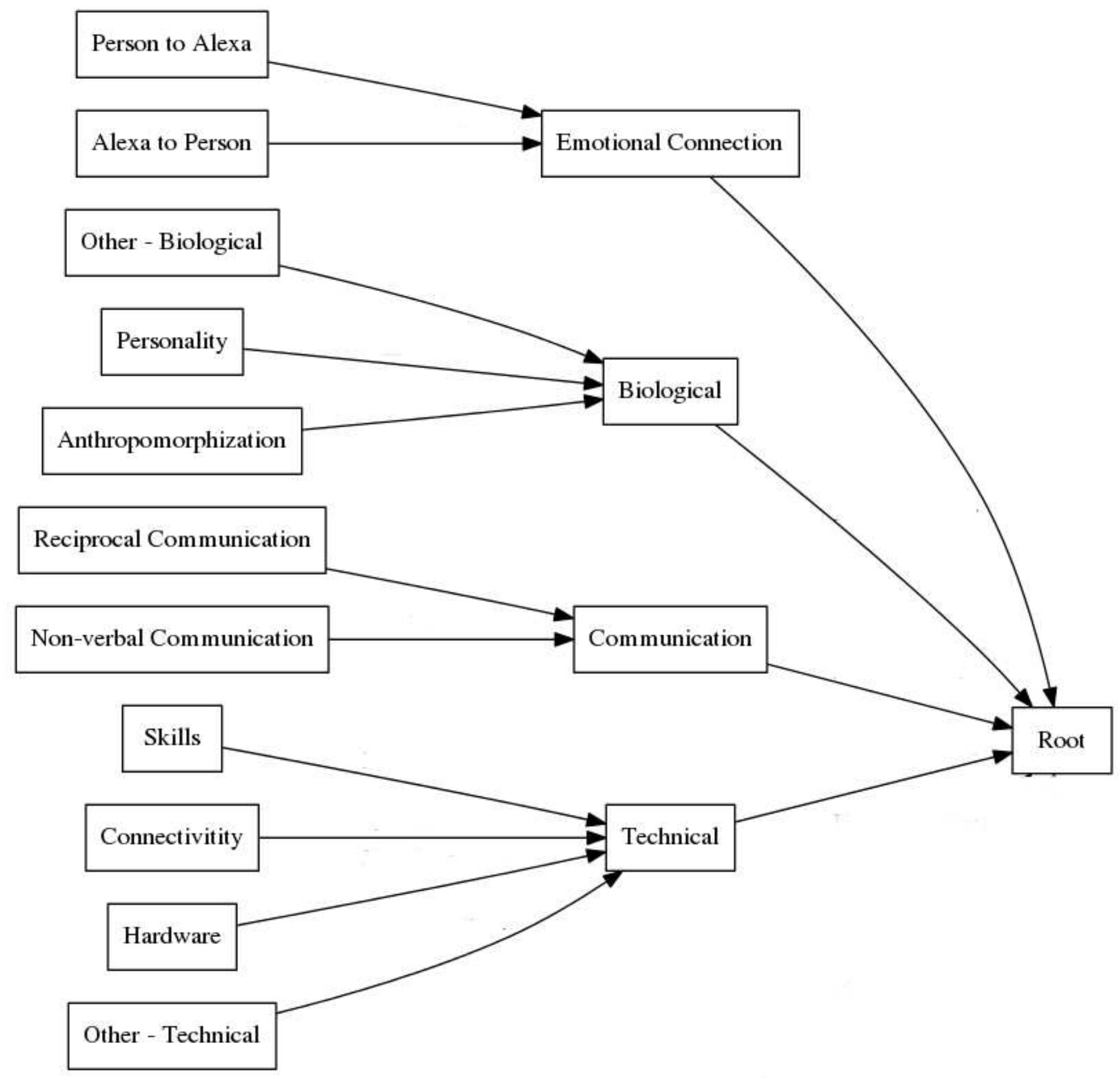

Figure 7

Graphical representation of top-level codes in the Alexa task. 


\begin{tabular}{|c|c|c|c|}
\hline$\bullet$ & Definition & Example & Subcode of \\
\hline Anthropomorphization & $\begin{array}{l}\text { Are users attributing human characteristics to Alexa, especially } \\
\text { in the way they address Alexa. Do they call the device her/she } \\
\text { versus it/device/thing. (only what they are calling Alexa) Always } \\
\text { have a code. Every line of code should have an } \\
\text { antrophomorphization code. }\end{array}$ & $\begin{array}{l}\text { Y: Alexa is overrated and she doesn't know anything you have to constantly } \\
\text { repeat yourself. N: Gave this as a gift hopefully they are enjoying it. } \mathrm{Y} / \mathrm{N} \text { : I love } \\
\text { playing with this device, she is so funny. }\end{array}$ & Biological \\
\hline Personality & $\begin{array}{l}\text { Are they describing the personality of Alexa? Is the device } \\
\text { funny, sassy, etc. }\end{array}$ & $\begin{array}{l}\text { I love speaking to Alexa, she is so funny. Sometimes she makes some snark } \\
\text { remarks that I do not expect. }\end{array}$ & Biological \\
\hline Other - Biological & $\begin{array}{l}\text { Gives Alexa any other human traits that does not to seem to fit } \\
\text { into any other human traits category }\end{array}$ & & Biological \\
\hline $\begin{array}{l}\text { Reciprocal } \\
\text { Communication }\end{array}$ & $\begin{array}{l}\text { Any other issues or situations where the owners have } \\
\text { conversations with Alexa. }\end{array}$ & $\begin{array}{l}\text { We do have words; from time to time when I ask a simple question and get "I } \\
\text { don't have an answer to that" or simple verbiage. After perhaps the third time of } \\
\text { rephrasing the question, i may get the right answer. }\end{array}$ & Communication \\
\hline $\begin{array}{l}\text { Non-verbal } \\
\text { Communication }\end{array}$ & $\begin{array}{l}\text { The light at the top of the device, shutting down instead of } \\
\text { giving a response. }\end{array}$ & $\begin{array}{l}\text { Some times I know Alexa is responding to me or thinking about how to respond } \\
\text { because the light at the top will be flashing. }\end{array}$ & Communication \\
\hline $\begin{array}{l}\text { Missing Alexa's } \\
\text { company }\end{array}$ & $\begin{array}{l}\text { The person using Alexa feels the absent of the device when } \\
\text { they are not using it }\end{array}$ & $\begin{array}{l}\text { When I am away from my home, I forget that Alexa is not there and I still try to } \\
\text { talk to her }\end{array}$ & Companionship \\
\hline $\begin{array}{l}\text { Alexa as Family } \\
\text { Member }\end{array}$ & Considers Alexa as part of the family & $\begin{array}{l}\text { Had the Echo and Alexa in our lives almost a year now and she's like a member } \\
\text { if the family. }\end{array}$ & Companionship \\
\hline Alexa as Friend & Considers Alexa a friend & Alexa is my new bestie. & Companionship \\
\hline
\end{tabular}

Figure 8

Detailed description of codes in the Alexa task.

\section{Apply Qualitative Coding Scheme to Barnstars}

Please review the coding schema in the system and do your best to apply the codes to the following sample text. First check the box for one or more top-level codes, then print all of the second level (or detail) codes that apply.

\section{Sample 1}

I hereby award you this barnstar for your extensive edits to [[East Brunswick]] and [[East Brunswick High School]]. Keep up the good work!

Check the box for one or more relevant top-level codes

Editing $\square$ Border Patrol $\square$ Collaborative Action $\square$ Meta Content $\square$ Social/Community $\quad \square$ Administrative $\square$ Misc/Unknown

Print the second level (or detail) codes that apply

Figure 9 
Example of a coding task. Participants were given instructions and two samples before beginning the task. They had the ability to freely use the system to complete all tasks. 